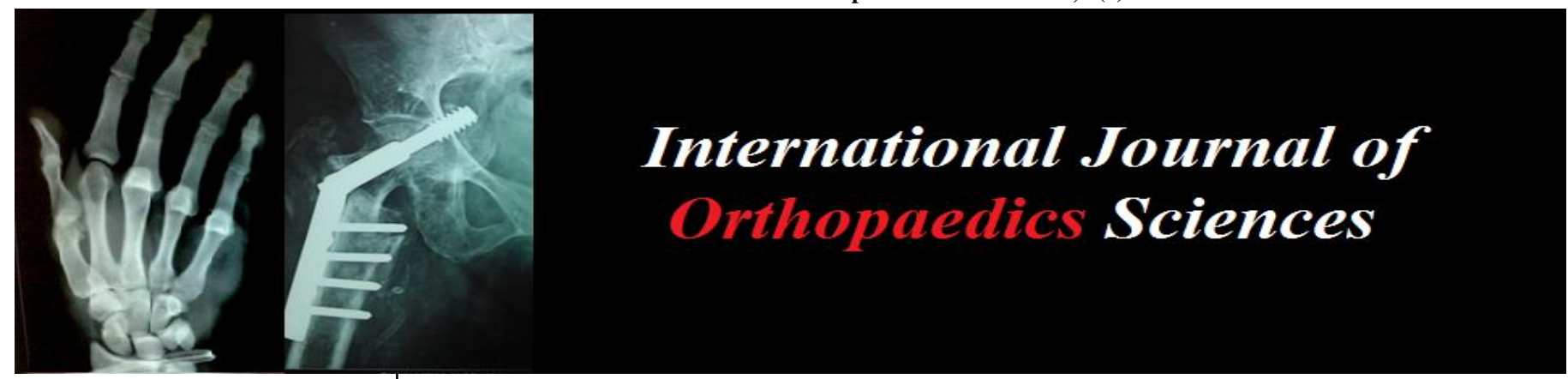

E-ISSN: 2395-1958

P-ISSN: 2706-6630

IJOS 2020; 6(3): 26-31

(C) $2020 \mathrm{IJOS}$

www.orthopaper.com

Received: 17-05-2020

Accepted: 19-02-2020

Dr. S Venkatesh Babu

Consultant Orthopaedic \&

Trauma Surgeon, Sri Sakthi

Hospital, Tirunelveli City, Tamil

Nadu, India

Corresponding Author:

Dr. S Venkatesh Babu

Consultant Orthopaedic \&

Trauma Surgeon, Sri Sakthi

Hospital, Tirunelveli City, Tamil

Nadu, India

\section{Safe knee surgery}

\section{S Venkatesh Babu}

DOI: https://doi.org/10.22271/ortho.2020.v6.i3a.2172

\section{Abstract}

At present, the surgical treatment has been standardised for the knee diseases and fractures. Though the surgical advances made in the implants and techniques, there are significant surgical complications. This review article emphasises the need of anatomical familiarity and safe operative skills for the surgeon.

Keywords: Safe, surgery, trauma, injury, knee, complications, artery, nerve

\section{Introduction}

At present, the surgical treatment of knee has been advised to most of its diseases and fractures. Though the surgical progress made in the implants and techniques of knee surgery, there are significant complications ${ }^{[8]}$.

Total knee arthroplasty is a safe, cost-effective treatment for alleviating pain and restoring function in patients with knee arthritis who are not responding to conservative treatment. It is a commonly performed procedure worldwide with a high rate of patient satisfaction. Despite this, complications may occur and can cause significant morbidity. Though the neurovascular complications are infrequent in knee surgery, its occurrence can become potentially disastrous for the affected lower limb. [Bernardi, Neagoe]. It is the duty of the surgeon to be aware of such risks in order to counsel the patient preoperatively and anticipate complications during surgery and in the post-operative period with prompt management ${ }^{[8]}$.

There are less invasive stabilisation system (LISS) and distal femoral nailing (DFN) for distal femoral fractures. With appropriate application, the distal femoral nail is suitable for all fractures of the distal third of femoral shaft including highly unstable bicondylar fractures without damage to the soft tissues and the knee joint ${ }^{[1]}$. There are no differences in outcome and complications with LISS and DFN in treating distal femoral fractures ${ }^{[4]}$.

The management of selected osteoporotic complex intra-articular fractures of the proximal tibia with compression bolts and intramedullary nailing offers specific advantages. In these type of fractures of the proximal tibia, treatment outcomes depend on the type of fracture as well as correct reduction and use of appropriate fixation. When accurate alignment, joint stability and well reduced articular surfaces are achieved, outcomes are very good ${ }^{[6,12]}$.

Many neurovascular complications that occur in knee surgery are preventable ${ }^{[2]}$. This article reviews and emphasise the need of anatomical familiarity and safe operative skills for the surgeon.

Principles of Safe Knee Joint Surgery ${ }^{[3,8,10,11,15,17,23,25,26]}$.

A thorough understanding of neurovascular anatomy, good preoperative and intraoperative planning with precise attention to the details of basic techniques and the equipment used for the procedure are important to the orthopaedic surgeon. It is also imperative, that the surgeon being aware of the complications recognise them at the earliest and initiate further evaluation and treatment as expeditiously as possible. 


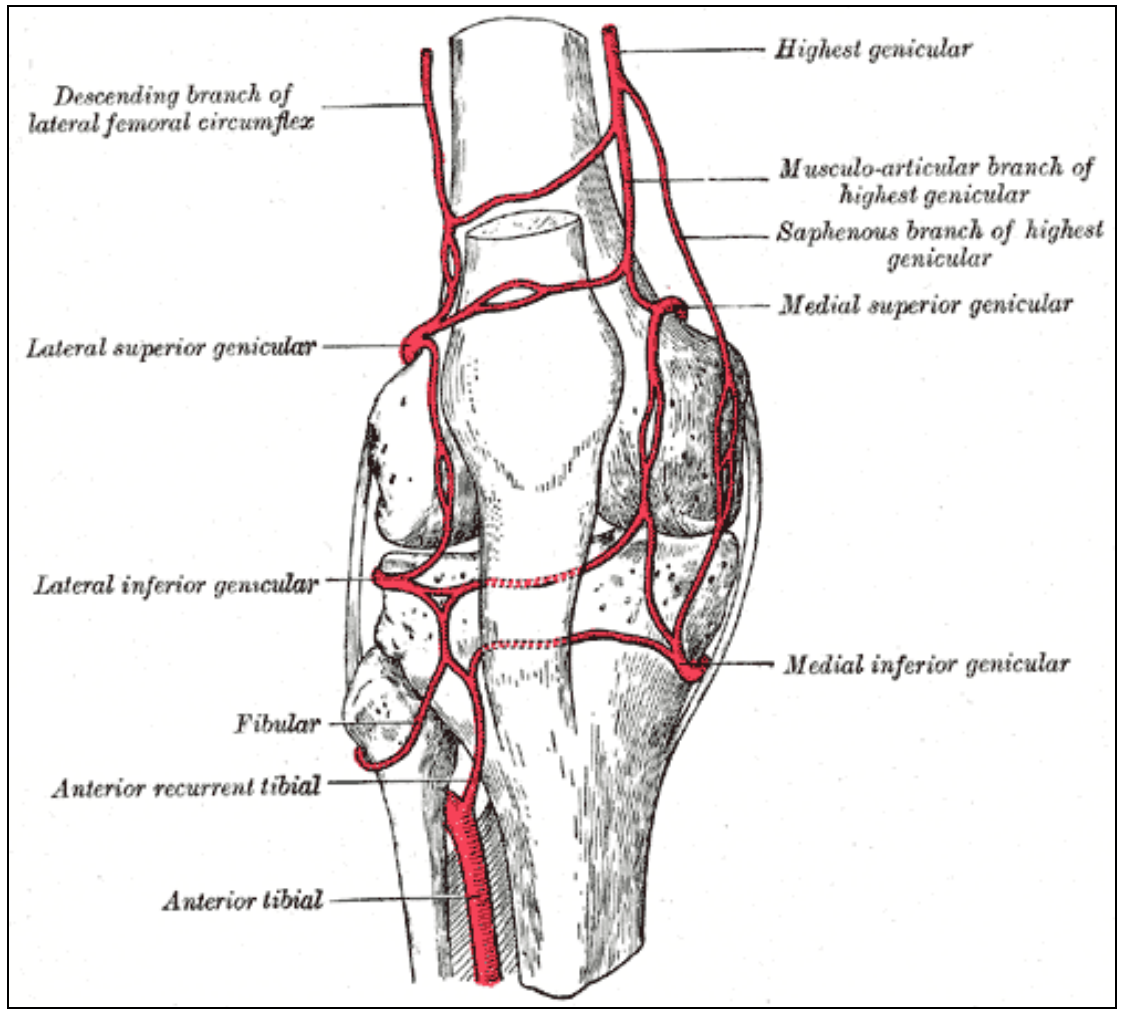

Fig 1: Knee Joint Vascular Anatomy

Safety facts in the Arthroscopic Surgery of the Knee Joint

- The articular cartilage of the knee may be damaged at two phases during arthroscopy: by the incision or by the forceful insertion of an arthroscope. If the incision is made cautiously, this problem will not occur. If we are meeting resistance while manipulating the arthroscope within the knee, then it is certain that we are damaging the articular cartilage.

- More posteriorly based incisions on the medial side may simply damage the articular surface of the medial femoral condyle, if performed unsighted. Therefore, it is recommended that more posterior medial or lateral incisions, if needed, should be made under direct arthroscopic control.

- The meniscus may be damaged by the scalpel or the arthroscope if the incisions are made too close to the joint line.

- Flexion of the knee in $90^{\circ}$ or the so- called" figure of four" position should be done when arthroscopic instruments approach the posterolateral corner of the knee to decrease the risk of nerve injury in cases of capsular penetration since the neurovascular structures are more distant from the knee capsule in this position.

Safety facts in the Medial Parapatellar approach of the Knee Joint

- The infrapatellar branch of the saphenous nerve often is injured during this approach.

- Patellar ligament has to be handled very gently, If the ligament becomes avulsed from its insertion on the tibia, it is hard to reattach.

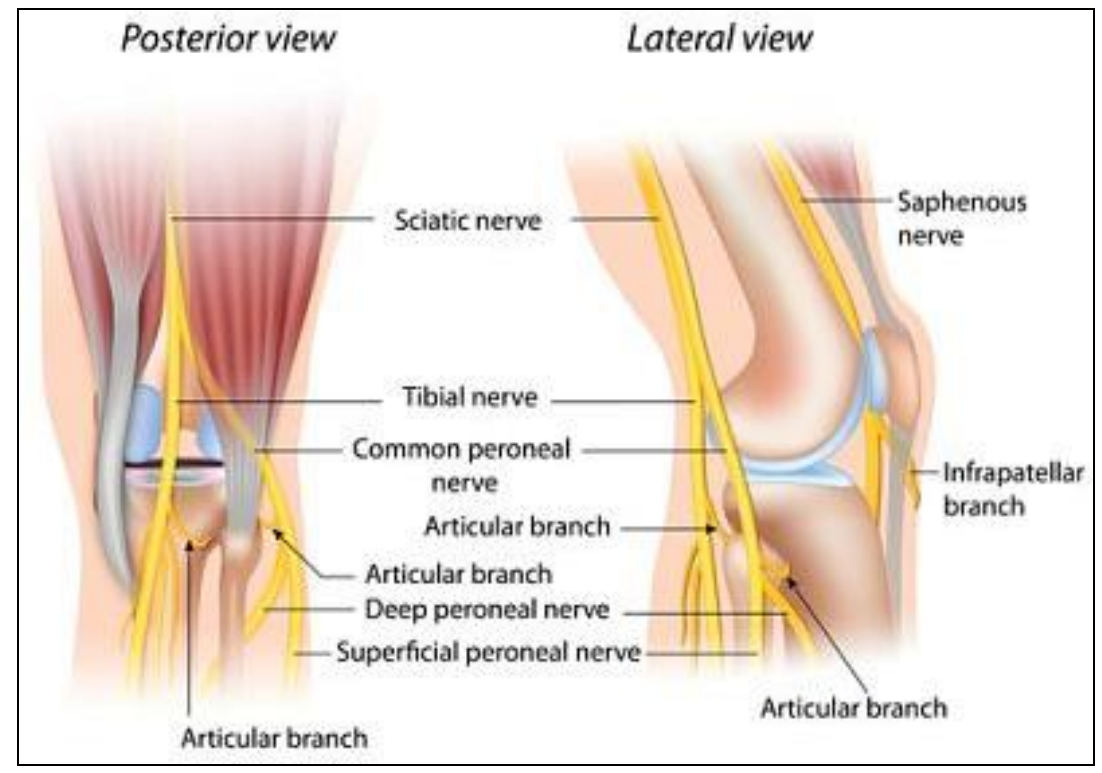

Fig 2: Knee Joint Neurological Anatomy 
Safety facts in the Medial Menisectomy of the Knee Joint

- The infrapatellar branch of the saphenous nerve may be injured here if the incision is extended farther inferiorly than $1 \mathrm{~cm}$ below the joint line.

- The popliteal artery is immediately behind the posterior joint capsule, any injury to the posterior joint capsule may damage the artery. If the knee is flexed, the posterior joint capsule falls away from the tibia and femur, taking the artery with it.

- The coronary ligament (the meniscotibial element of the deep medial ligament) connects the periphery of the meniscus with the joint capsule and tibia, and may be damaged if the incision through the synovium is made at the joint line.

- Incisions made too far posteriorly may injure the superficial medial ligament (the tibial collateral ligament) as it runs from the medial epicondyle of the femur to its insertion on the tibia under cover of the pes anserinus.

- The fat pad occupies varying amounts of the anterior portion of the knee joint and should not be damaged. Injury may produce adhesions within the joint and may compromise the blood supply of the patella.

- The medial meniscus may be incised accidentally during the opening of the synovium unless the knee joint is entered well above the joint line.

Safety facts in the Medial approach of the Knee Joint and its supporting structures

- The saphaneous nerve emerges between the gracilis and sartorius muscles and runs with the long saphenous vein. It provides sensation for some of the non-weight-bearing portions of the foot and should be preserved.

- The saphaneous vein appears in the posterior corner of the superficial dissection.

- The medial inferior genicular artery curves around the upper end of the tibia. It may be damaged when the medial head of gastrocnemius muscle is lifted off the posterior capsule.

- The popliteal artery lies against the posterior joint capsule in the midline and is adjacent to the medial head of the gastrocnemius muscle. Upmost care must be taken to avoid injuring the vessel during separation of the gastrocnemius from the joint capsule.

\section{Safety facts in the Lateral Menisectomy of the Knee Joint}

- The lateral meniscus may be damaged if the synovium is incised too close to the joint line.

- The lateral inferior genicular artery runs around the upper part of the tibia. The artery lies next to the peripheral attachment of the lateral meniscus; it may be injured if the meniscus is detached along with a portion of the capsule during meniscectomy, leading to massive haemarthrosis.

- The superficial lateral ligament (Fibular collateral ligament) limits posterior extension at the incision. If it is injured and not reconstructed may lead to lateral instability.

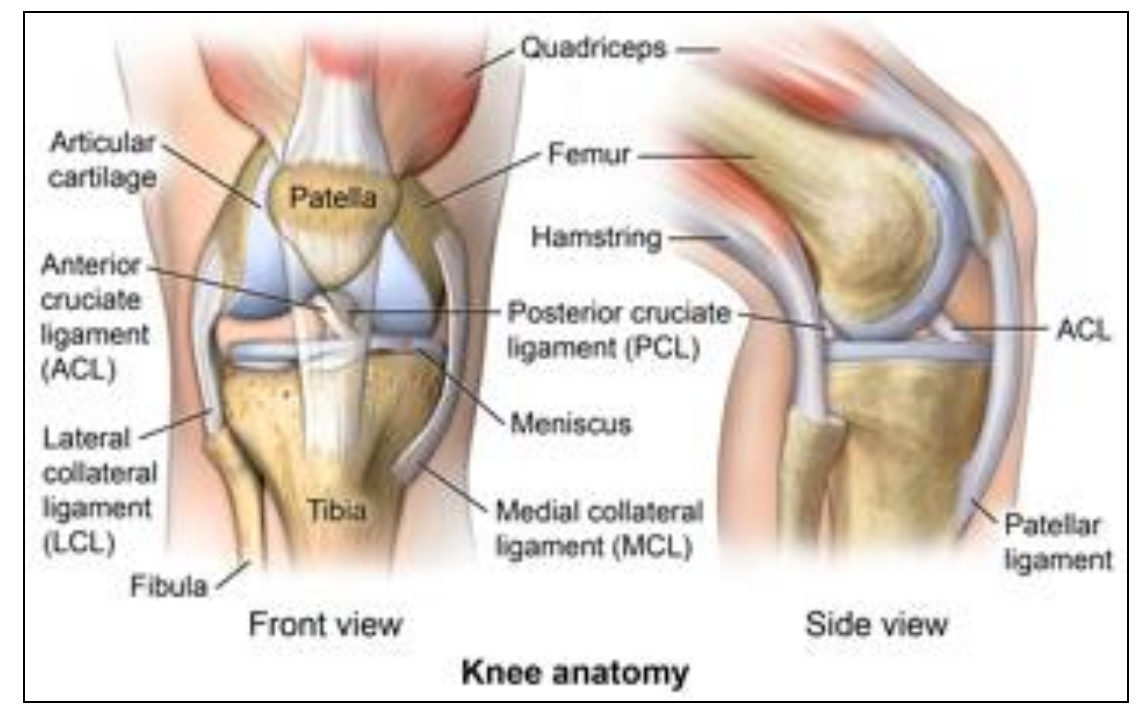

Fig 3: Knee Joint with its ligaments

\section{Safety facts in the Lateral approach of the Knee Joint}

- The common peroneal nerve is the structure most at risk during this approach. It lies on the posterior border of the biceps tendon and must be found early in the approach, as the supporting structures of the lateral side of the knee getting dissected, the nerve has to be identified and protected well.

- The lateral superior genicular artery runs between the lateral head of the gastrocnemius muscle and the posterolateral capsule and requires ligation for full exposure of the posterolateral corner.

- The popliteus tendon is at risk as it travels within the joint before it reaches to the posterior aspect of the meniscus and the femur.Taking care while opening the posterior half of the knee joint capsule to avoid cutting the tendon.

- The lateral meniscus or its coronary ligament may be incised accidentally, if arthrotomy is performed too close to the joint.

\section{Safety facts in the Posterior approach of the Knee Joint}

- The medial sural cutaneous nerve, which lies lateral to the small saphenous vein, may be damaged as it travels beneath the deep fascia of the calf.Incising the deep fascia of the calf medial to the vein therefore, will preserve the nerve.

- The tibial nerve may be damaged in the popliteal fossa and the common peroneal nerve also is susceptible to damage in the politeal fossa. Hence much care has to be given while dissecting. 
- The small saphenous vein and popliteal vessels can be injured during the deep dissection.

Safety facts in the Lateral approach of the distal femur for Anterior Cruciate Ligament Surgery

- The peroneal nerve can be injured, if the surgical plane is strayed out of the posterior side of the biceps femoris muscle.

- The lateral superior genicular artery must be take care while incising.

- $\quad$ The popliteal artery may be injured if the surgical plane doesnot remain subperiosteal. As the intercondylar notch is felt, do bend the knee to $90^{\circ}$ to allow the popliteal artery to fall posteriorly with the joint capsule.

Safety facts in the Minimal Invasive surgical (MIS) approaches of LISS, Nailing and knee replacement surgery

- The Minimal invasive approach for extra- and intraarticular distal femoral fractures is dissimilar.

- In extra-articular fractures, a skin incision from Gerdy's tubercle about $80 \mathrm{~mm}$ in proximal direction is made. Then, splitting the iliotibial tract in the direction of the fibres is made. Openning the space between the lateral vastus and the periosteum is done. Distally, the lateral vastus muscle inserts mainly on the femoral ridge. There are no muscle insertions on the lateral periosteum or bone. The internal fixator (Less Invasive Stabilisation system-LISS) can be inserted into the space between the periosteum and the muscle.
- In intra-articular fractures, an anterolateral arthrotomy providing good control of the reduction is recommended. This arthrotomy also allows a subsequent insertion of the LISS fixator and can be used to insert lag screws from medially.

- The most common errors in the LISS while treating distal femoral fractures are related to inadequate reduction of the fracture. Intraoperative proper usage of fluoroscopy and correct application of principles of LISS as advised by the creators of LISS will yield excellent results.

- There are several MIS techniques have been described for knee replacement surgery which minimize quadriceps damage and avoid patellar eversion.

- The mini-arthrotomy technique uses a smaller medial parapatellar arthrotomy than a traditional medial parapatellar arthrotomy does.

- The mini-midvastus technique has an arthrotomy that extends $2 \mathrm{~cm}$ or more into the vastus medialis muscle.

- The medial quad-sparing approach uses a more medial incision and avoids a quadriceps sparing arthrotomy.

- The direct lateral approach utilizes an 8 to $10 \mathrm{~cm}$ incision from below Gerdy's tubercle to the lateral epicondyle lateral to the patella.

- The optimal entry point for retrograde femoral nailing (DFN) is $1.2 \mathrm{~cm}$ anterior to the femoral origin of the posterior cruciate ligament and centered in the intercondylar sulcus provides the optimal balance of fracture reduction and knee joint sparing.It may be difficult to target this site with a percutaneous technique and may require direct visualization of the intercondylar sulcus for ideal nail placement.

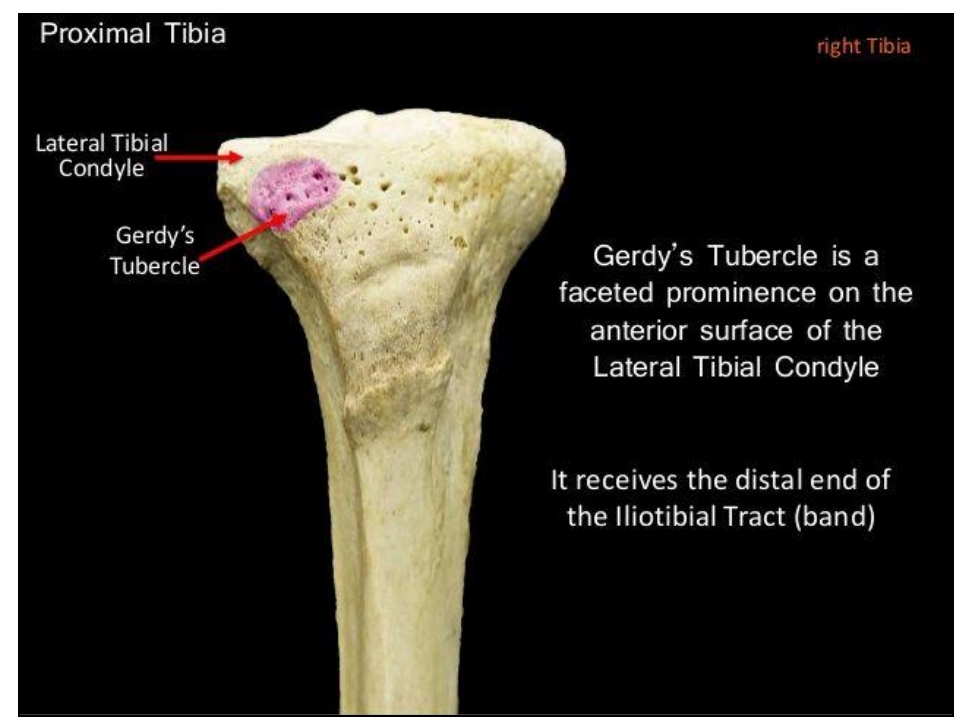

Fig 4: The Proximal tibia and Gerdy's tubercle

\section{Discussion}

Since its inception in medical practice, arthroscopy has been considered as the standard and safe method in diagnosis and treatment of numerous conditions of knee with fewer complications. However, latest reports on knee arthroscopy and its overall complications are in rise with incidences between $0.27 \%$ and $4.7 \%$. There are major iatrogenic vascular trauma which caused severe morbidity to the patients been reported. The meniscus arthroscpic surgery had faced more vascular injuries than other ligaments. High index of suspicion is warranted with immediate treatment in these injuries ${ }^{[14,19]}$.

The typical valgus and varus bending mechanism can result in a corresponding lateral or medial plateau split fracture with a varying degree of articular surface depression. This situation leads to axis malalignment and pathological load distribution, which ultimately causes poor functional outcome and posttraumatic osteoarthritis ${ }^{[24]}$. Concomitant ligament disruption, meniscus or cartilage damge will further weaken clinical results. Non-operative treatment may be considered for nondisplaced intra-articular fractures of the lateral tibial condyle. Intra-articular joint displacement more than $2 \mathrm{~mm}$, Metaphyseal-diaphyseal translation of $>1 \mathrm{~cm}$, lateral tilting of the plateau $>5^{\circ}$, Condylar widening $>5 \mathrm{~mm}$, Angular deformity of $>10^{\circ}$ in either the coronal (varus-valgus) or sagittal plane (tibial slope), associated compartment 
syndrome, associated ligamental injury requiring repair, associated fractures of the ipsilateral tibia or filbula, open fractures, all fractures of the medial condyle should be reduced and fixed operatively ${ }^{[20]}$. In the complex high energy proximal tibial fractures involving metadiaphyseal segment, the restoration of the limb axis and length, accurate intraarticular reduction, further avoidance of soft tissue injury, is the most crucial step. These fractures are associated with extensive soft tissue damage even in closed fractures. The management requires excellent soft tissue handling and fracture reduction skills to avoid iatrogenic complications ${ }^{[17}$, 18]

Accurate anatomical reduction of the knee articular surface, restoration of functional anatomy and stable fixation are mandatory for an optimal knee function after distal femoral fractures. Failure to achieve them, due to the pattern of the fracture or to improper fixation is followed by major functional deficit, thus delaying the patient's recovery ${ }^{[16]}$.

The mortality rate of Total knee replacement was reported to be greater in low volume institutions than in high volume institutions $(0.73 \%$ vs $0.43 \%)$. Around $0.5 \%$ to $12 \%$ of patients who had knee replacement develop deep infection around the prosthesis: periprosthetic knee infection and also very low incidence of infection after knee arthroscopy ${ }^{[5,8]}$. This challenge and its treatment can be life-changing for patients and there is a need for greater support throughout treatment and leads to lengthy recovery. Patients who develop periprosthetic knee infection will experience profound disruption to their life course ${ }^{[21,22]}$. It is highly recommended to implement antibiotic stewardship programme and implementations of safety bundles in all the health establishments today to prevent and manage the Surgical Site Infection effectively $[11,15,23,25,26]$.

The advantages of minimally invasive surgery in total knee replacement are purported to be improved wound cosmesis, faster recovery time and subsequent decreased length of hospital stay and improved function compared to standard total knee arthroplasty approach. A substancial learning curve may exist with minimal invasive techniques, which may be unacceptably long for surgeons performing low volumes of knee replacement surgery however the cemented total knee arthroplasty using a conventional medial parapatellar approach without computer navigation remains the gold standard ${ }^{[8]}$.

Although the incidence of Nerve injuries following knee surgeries is low, there is increased occurrence in the diabetic population. The common peroneal nerve injury is commonest in the knee arthroplasty and arthroscopy. However the saphaneous nerve may also get injured significantly in the arthroscopy. (0.3-10\%). Potential mechanisms of iatrogenic nerve injury include nerve puncture, sutures tied over the nerve or nerve capture and tension placed on the nerve or nerve tethering. The total incidence of neurovascular after total knee replacement has been reported between $0.9 \%$ and $1.3 \%$. The common peroneal nerve is the commonest injured nerve and the popliteal artery is the commonest major vessel at risk. The injury has to be identified and promptly treated. Medico-legally, the identified negligence is often the faulty management of the palsy postoperatively, rather than the actual occurrence of the nerve injury ${ }^{[7,8,9]}$.

Orthopaedic surgeons should be aware that all kind of complications including neurovascular and other vital structures injuries could be encountered after knee surgeries and should be able to recognise its clinical picture instantaneously.

\section{Conclusion}

At present, the surgical treatment has been advised for the knee diseases and fractures. Though the surgical advances made in the implants and techniques, there are significant surgical complications. It is highly recommended that the operating surgeon should have the good anatomical knowledge and safe operative skills.

\section{References}

1. Grass et al. Retrograde Locking Nail Osteosynthesis of Distal Femoral Fractures with the Distal Femoral Nail (DFN), Unfallchirurg. 2002; 105(4):298-314.

2. Kim et al. Neurovascular Complications of Knee Arthroscopy. The Am J Sports Med. 2002; 30(4):619-29.

3. Krupp et al. Optimal Entry Point for Retrograde Femoral Nailing. J Orthop Trauma. 2003; 17(2):100-5.

4. Markmiller et al. Femur-LISS and Distal Femoral Nail for fixation of distal femoral fractures: Are there Differences in Outcome and complications? Clin Orthop Relat Res. 2004; (426):252-7.

5. Reigstad et al. Complications in Knee Arthroscopy, Knee Surg Sports Traumatol Arthrosc. 2006; 14(5):473-7.

6. Pasa et al. Treatment of Intra-articular Proximal Tibial Evaluation of Two-to Seven-year Follow up, Acta Chir Orthop Traumatol Cech. 2007; 74 (5):336-41.

7. Estrella et al. Isolated Extensor Hallucis Longus Paralysis after Knee Arthroscopy: A Case Report, The Foot \& Ankle Journal. 2008; 1(10):1-4.

8. Cheung et al. Complications of total knee arthroplasty, Current Orthopaedics. 2008; 22:274-283.

9. Yacub et al. Nerve Injury in Patients following Hip and Knee Arthroplasties and Knee Arthroscopy. Am J Phys Med Rehabil. 2009; 88(8):635-91.

10. Stanley Hoppenfeld et al. Surgical Exposures in Orthopaedics, The Anatomic Approach, Fourth Edition, Lippincott Williams \& Wilkins, 2009.

11. WHO. Guidelines for safe surgery: safe surgery saves lives, 2009.

12. Garnavos et al. The Management of Complex Fractures of the Proximal Tibia with Minimal Intra-Articular Impaction in Fragility Patients using Intramedullary Nailing and Compression Bolts, Injury. 2011; 42(10):1066-72.

13. Pakula et al. Errors in Treatment of fractures of distal femur by LISS method (Less Invasive Stabilisation system) - Single Centre Experience, Ortop Traumatol Rehabil. 2014; 16(3):275-84.

14. Neagoe et al. Major vascular injuries complicating knee arthroscopy, Videosurgery Miniiv. 2015; 10(2):266-274.

15. Antimicrobial Stewardship: systems and processes for effective antimicrobial medicine use, NICE Guidelines, UK, 2015.

16. Lupescu et al. Treatment Options for distal Femoral Fractures, Medical Journal of Clinical Medicine. 2015; 10(2):117-122.

17. Wu et al. A Useful strategy for proximal tibial fractures (AO/OTA type 41-C) with diaphyseal involvement. Int J Clin Exp Med. 2015; 8(8):13455-13463.

18. Khatri et al. Complications in the management of closed high-energy proximal tibial plateau fractures, Chinese Journal of Traumatology. 2016; 19:342-347.

19. Bernardi et al. Transient Common Peroneal and Tibial Nerve Palsy Following Knee Arthroscopy for the Treatment of Discoid Lateral Meniscus, Case Report, Joints. 2017; 5:118-120. 
20. Raschke et al. Proximal tibia fractures, EOR. 2017; 2:241-249.

21. Lu et al. Infection after teotal knee arthroplasty and its gold standard surgical treatment: Spacers used in two stage revision arthroplasty, Intractable \& Rare Diseases Research. 2017; 6(4);256-261.

22. Mallon et al. Infected knee replacement: a qualitative study of impact of periprosthetic knee infection, BMC Musculoskeletal Disorders. 2018; 19(352):1-10.

23. Seligman et al. Preventing Surgical Site Infection after Cesarean Delivery-The Anaesthesia Professional's role, APSF News Letter, 2018, 25-27.

24. Aurich et al. Communited Intraarticular Fractures of the Tibial Plateau lead to Posttraumatic Osteoarthritis of the Knee: Current Treatment Review. Asian J Surg., 2018; 41(2):99-105.

25. Surgical site infections: prevention and treatment, NICE Guidelines, UK, 2019.

26. Antimicrobial stewardship programmes in health care facilities in Low and middle income countries, A World Health Organisation (WHO) Tool Kit, 2019. 